\title{
Campylobacter Gastroenteritis
}

National Cancer Institute

\section{Source}

National Cancer Institute. Campylobacter Gastroenteritis. NCI Thesaurus. Code C35162.

Gastroenteritis resulting from an infection with Campylobacter. 